\title{
Can digitally collected PROMs as intervention for post-treatment adaption improve cost effectiveness: study protocol for a randomized controlled trial
}

\section{David Kuklinski ( $\sim$ david.kuklinski@tu-berlin.de)}

Technische Universitat Berlin https://orcid.org/0000-0002-7092-7480

\section{Laura Oschmann}

Technische Universitat Berlin

\section{Christoph Pross}

Technische Universitat Berlin

\section{Reinhard Busse}

Technische Universitat Berlin

\section{Alexander Geissler \\ Technische Universitat Berlin}

\section{Study protocol}

Keywords: Health care policy, PROM, Quality of care, TKR, THR, Value-based health care

Posted Date: February 27th, 2020

DOI: https://doi.org/10.21203/rs.2.24694/v1

License: (c) (i) This work is licensed under a Creative Commons Attribution 4.0 International License. Read Full License

Version of Record: A version of this preprint was published at Trials on April 9th, 2020. See the published version at https://doi.org/10.1186/s13063-020-04252-y. 


\section{Can digitally collected PROMs as intervention for post- treatment adaption improve cost effectiveness: study protocol for a randomized controlled trial}

\section{Corresponding author:}

David Kuklinski

Technical University Berlin, Straße des 17. Juni 135, 10623 Berlin

David.kuklinski@tu-berlin.de

\section{Supporting authors:}

Laura Oschmann

Technical University Berlin, Straße des 17. Juni 135, 10623 Berlin Oschmann@tu-berlin.de

Dr. Christoph Pross

Technical University Berlin, Straße des 17. Juni 135, 10623 Berlin Christoph.pross@tu-berlin.de

Prof. Dr. Reinhard Busse

Technical University Berlin, Straße des 17. Juni 135, 10623 Berlin rbusse@tu-berlin.de

PD Dr. Alexander Geissler

Technical University Berlin, Straße des 17. Juni 135, 10623 Berlin A.geissler@tu-berlin.de 
Title: Can digitally collected PROMs as intervention for post-treatment adaption improve cost effectiveness: study protocol for a randomized controlled trial

Authors: (1) David Kuklinski (2) Laura Oschmann (3) Christoph Pross (4) Reinhard Busse (5) Alexander Geissler

Background: The number of total knee and hip replacements (TKR/THR) has been increasing noticeably in high-income countries, such as Germany. Especially the number of revisions is expected to rise due to higher life expectancy and procedures performed on younger patients, impacting budgets of the health care systems. Quality transparency is the basis of holistic patient pathway optimization. Nevertheless, a nation-wide cross-sectoral assessment of quality from patient perspective does not yet exist. Several studies have shown that the use of patient-reported outcome measures (PROMs) is effective in measuring quality and for monitoring post-treatment recovery. For the first time in Germany, we test whether early detection of critical recovery paths using PROMs after TKR/THR improves the quality of care in a cost-effective way and can be recommended for implementation into standard care.

Methods/design: The study is a two-arm multi-center patient-level randomized controlled trial. Patients from nine hospitals are included in the study. Patient-centered questionnaires are employed to regularly measure digitized PROMs of TKR/THR patients from the time of hospital admission until 12 months post-discharge. An expert consortium has defined PROM alert thresholds at 1, 3, and 6 months to signal critical recovery paths after TKR/THR. An algorithm alerts study assistants if patients are not recovering in line with expected recovery paths. The study assistants then contact patients and their physicians to investigate and, if needed, adjust the post-treatment protocol. By adding sickness funds' claims data, the cost-effectiveness of the intervention can be analyzed.

Discussion: The study is expected to deliver an important contribution to test PROMs as an intervention tool and examine the determinants of high-quality endoprosthetic care. Depending on a positive and cost-effective impact, the goal is to transfer the study design into standard care. During the trial design phase, several insights have been discovered, with opportunities for efficient digital monitoring limited by existing legacy care models. Digitalization in hospital processes and the implementation of digital tools still represents challenges for hospital personnel and patients. Furthermore, data privacy regulations and the separation between the in- and outpatient sector are additional roadblocks to effectively monitor and assess quality along the full patient pathway.

Trial registration: German Clinical Trials Register: DRKS00019916. Registered 26 November 2019 - retrospectively registered, https://www.drks.de/drks_web/navigate.do?navigationId=trial.HTML\& TRIAL_ID=DRKS00019916.

Keywords: Health care policy, PROM, Quality of care, TKR, THR, Value-based health care 


\section{Background}

Total knee and hip replacements are among the most frequent and increasing surgeries in high-income countries such as Germany. They are considered to be effective but also highly invasive procedures to treat osteoarthritis in the knee and hip. In 2016 in Germany, there were more than 187,000 procedures (230 per 100,000 population) for total knee replacements (TKR) - a 38\% increase compared to 2006 and 233,000 procedures (280 per 100,000 population) for total hip replacements (THR) - a $17 \%$ increase compared to 2006 (1-3). Moreover, the number of primary as well as revision procedures is expected to rise steadily due to increased life expectancies and procedures performed on younger patients (2). This development has a significant impact on the budgets of health care systems. Measuring patient-reported quality helps to generate transparency, to evaluate treatments, and as a consequence to optimize patients' pathways (indication, procedure, recovery), leading to enlarged revision horizons and a decreased number of revisions (4).

Quality of joint replacement in Germany is measured and monitored by several mandatory or voluntary initiatives. They concentrate on the collection and analyses of clinical quality indicators and administrative data, as well as the adherence to structural and processual standards and the interpretation of medical documentation (5). These quality initiatives are mostly limited to the acute inpatient sector (6). A nation-wide standardized and cross-sectoral assessment of quality from patient perspective does not exist yet. As a result, the improvement of arthroplasty-relevant problems like post-operative joint functionality, pain or constraints in daily live cannot be assessed by the existing quality measurement tools. However, an improvement in those quality dimensions defines treatment success (7) and significantly affects the patient's utility (8). Consequently, a recent study in the United Kingdom concluded that for THR patients, quality measured by patient-reported outcomes (PROMs) significantly affects patient's utility in form of willingness to travel and hospital demand positively. In contrast, existing quality indicators such as mortality rates or readmission rates have either only a small positive or even insignificant effect (8).

Furthermore, increased transparency of the patients' recovery process using PROMs initiates a direct feedback mechanism between physician and patient, thus optimizing surgeries $(9,10)$ and best practice sharing (7). At present this is barely given. After rehabilitation, patients will seldom return to the hospital for follow-up visits but rather see their family physicians or outpatient specialists. Unfortunately, communication channels between hospitals and follow-up physicians are often not present, preventing any feedback mechanism regarding their patients' recovery process (11).

For example, the effects of improved medical quality through continuous monitoring patients after surgery using PROMs has been concluded in a study with cancer patients. The study shows that during 
routine treatment, constant monitoring and early detection of critical recovery paths via PROMs significantly improved survival rates and decreased the number of emergency visits $(12,13)$.

Our study is designed to go beyond testing the effectiveness of PROMs as a quality measurement instrument. For the first time in Germany, we want to test whether an early detection of critical recovery paths after TKR/THR via PROMs and change of post-treatment pathways improves clinical quality indicators such as readmissions and patient-centered quality measures such as health-related quality of life and joint functionality, and in turn, leads to a decrease of health care costs. The study focuses primarily on two specific questions: (1) Can the use of PROMs after TKR/THR impact the patient pathway in a positive and cost-effective manner? (2) Can we recommend the implementation of PROMs into the standard care and what are best practices to follow? 


\section{Methods / Design}

\section{Overall study design}

This study is a two-arm multi-center patient-level randomized controlled trial (RCT). Patient-centered questionnaires are employed digitally to regularly measure PROMs of patients with TKR and THR from the time of hospital admission until 12 months after discharge. With support of an expert consortium of physicians, PROM threshold alert values at 1, 3, and 6 months have been defined to signal critical recovery of patients after TKR/THR. By the help of an algorithm, patients and posttreatment physicians are alerted to intervene in case of critical PROM values.

By adding cross-sectoral insurance claims data (resource consumption: drugs, physiotherapy, specialist visits, complications, infections, hospital stays) on patient level, the study examines the effect of PROM-based monitoring of patients up to 12 months post-surgery on the clinical as well as patientreported outcomes and its cost-effectiveness.

\section{Ethics, consent, permissions \& funding}

The study is funded by the Innovation Fund of the German Federal Joint Committee (G-BA) in the stream 'Care models with comprehensive and measurable results and process responsibility'. The funding period is set between April 1st, 2019 and March 31st, 2023. The study will be conducted in accordance with the Declaration of Helsinki. The study was primarily approved by the Charités Ethic Committee, Berlin (EA4/169/19). The other responsible ethical review committees of participating hospitals (Medical Chamber Hamburg, Medical Chamber Schleswig-Holstein, Hannover Medical School, Friedrich-Schiller University Jena, and Medical Chamber Brandenburg) agreed with the decision. The study is registered at the German Clinical Trials Register (DRKS) under trial number DRKS00019916. All potentially eligible participants will be approached to offer their informed consent to participate in the study. The current protocol is version 1, dated January $15^{\text {th }} 2020$. Any changes in the study design will be communicated to all project partners, including hospitals.

\section{Study setting}

The study has started on October $1^{\text {st }} 2019$ and will last for two years, whereas patient recruitment will end after one year, on September $30^{\text {th }} 2020$. The second year will concentrate on follow-ups and corresponding interventions. After two years, the study will be evaluated by an independent and impartial research institute (aQua Institute). Study centers are the nine participating hospitals. These hospitals are located around Germany (seven different federal states) and are among Germany's leading endoprosthetic centers. Hospitals differ in their type, including university hospitals, specialized endoprosthetic centers, full service providers, and specialist outpatients clinics. 
Based on historical hospital case volumes for elective TKR/THR to estimate expected workload, up to two study assistants are employed at each center - funded by means of the Innovation Fund - to operationalize the study design in each center. Before the start of the project, these study assistants were trained thoroughly in workshops to be in line with the specifications of the study design. The study assistants are responsible for all study-related activities during the patients' hospital stay, for follow-up as well as the initiation and documentation of interventions and a complete final documentation of the participant after 12 months.

The study assistants and patients utilize a software interface provided by the HRTBT Medical Solutions $\mathrm{GmbH}$, a digital solutions provider for hospitals, to document patient enrollment, key procedure aspects and the patient-reported outcomes. Patients' health procedure and claims data along the care pathway is accessed via the participating German sickness funds BARMER and BKK, both part of the social health insurance scheme (14). These insurance claims data will be extracted for four years (2018-2021) for those patients insured in one of these funds and having consented to study design and data sharing.

\section{Sample size}

The estimation of the study's sample size is based on historical case volumes (identified by procedure codes: OPS 5-820, 5-822) of the nine study hospitals from 2018. Hence, we expect around 17,500 patients to be eligible in the 12-month recruiting time frame. This figure already excludes emergency patients and minors. Furthermore, HRTBT Medical Solutions GmbH's prior field studies suggest that around $15 \%$ of patients reject participation, and around $30 \%$ of the remainder will not finalize all followup surveys, despite reminders and active follow-up efforts. Consequently, we expect to reach a sample size of around 10,500 participants having completed all follow-ups. This number is not yet adjusted for people without direct or indirect access to the internet or other technical constraints but increasing case volumes compared to 2018 are expected to balance this effect.

Furthermore, it is expected that insurance claims data for patients insured in the two participating sickness funds (approx. 2,700 patients) can be obtained, resulting in a sample size of 2,700 for our primary endpoint. If we assume a statistical difference in effect between intervention and control group of 0.15 standard deviations, an error probability of less than $5 \%$ and a power of $80 \%$, a sample size of only around 1,400 patients with insurance claims data is required for analysis.

\section{Eligibility criteria}

Eligible for this study are all patients with primary elective TKR and THR above the age of 18 having a scheduled surgery or pre-surgery visit between October 2019 and September 2020. Exclusion criteria for participation are emergencies (e.g., femoral fracture), patients with ASA classification 4-6 (4 patient with a life-threatening disease, 5 - moribund patient who is unlikely to survive without surgery, 6 - deceased patient with confirmed brain death, or organ donor), and patients younger than 18 years. Additionally, patients without direct or indirect access to an e-mail account are excluded from the study 
due to practical reasons. E-mail addresses from relatives are accepted. All patients who deny participation will not be included in the study.

\section{Informed consent}

All patients with a primary elective TKR or THR will be approached by their responsible study assistants for a patient briefing once they have been scheduled a surgery in the corresponding hospital. Patients will then be informed by either the study assistant or the responsible physicians of the study and its benefits, risks and design. Additionally, they are handed a comprehensive study information document. Before including the patients, they are required to sign an informed consent for their study participation (Supplement 1). The informed consent allows for contacting the patient via e-mail, post, and telephone and for processing and linking the patient's data from different sources during and after the study.

There are two additional consent forms that the patients are asked to sign: (1) the consent form for processing their hospital administrative data and (2) the consent form for processing their insurance data in case that the patient is contracted with one of the participating sickness funds. Both of these consent forms are optional and are not required to participate in the study.

Patients will be free to withdraw from either one of the three consents without stating a reason. If the patients withdraw from the consent for participation, then all their data will be deleted. Patients are allowed to participate in other studies as long as these are not interfering with our study.

\section{Participant timeline}

Each participant will be participating in the study for a maximum of 12 months plus the duration of the hospital stay, starting with the admission day, followed by the inpatient stay, the discharge and postdischarge period. The participant will have to answer two questionnaires during the hospital stay, at admission and discharge. At discharge, the randomized allocation into intervention and control group takes effect, and the participant is followed upon with electronic questionnaires either one or four times in the 12 months post-hospital discharge. With the final questionnaire after 12 months, the study will conclude for each participant regardless of allocation. The timeline for the participants can be seen in Figure 1.

\section{Baseline assessment}

Baseline assessment takes place for all eligible participants at the day of admission into the hospital. The timing slightly differs between the study centers. Three study centers perform the baseline assessment during their pre-surgery examination days, this can be up to six weeks before surgery. After the informed consent, the participant will be included in the study by providing name and e-mail address. In the next step, while waiting for the physician's appointment, the participant is given a tablet 
to respond to a 10-15 minutes questionnaire, consisting of basic demographic, comorbidity information and selected PROM sets.

The overall questionnaire is based on the ICHOM Standard Set for Hip and Knee Osteoarthritis consisting of the EQ-5D-5L, HOOS-PS/KOOS-PS and an analogue pain scale. This set was refined by adding the PROMIS Depression and Fatigue sets, as studies show that the recovery process is also affected by patients' mental conditions (15). When selecting the PROM sets, three criteria were of utmost importance: Firstly, the questionnaire should be brief to include it into the hospital's daily activities. Secondly, the sets should be renowned and proven by literature. Finally, the combined set of PROMs should cover all important patient-related aspects of the osteoarthritic condition and the elective TKR and THR procedures - health-related quality of life, functionality, pain, and mental condition.

At discharge from the hospital, the participant once again is handed a tablet to complete, by himself or by help of the study assistant, a mostly identical questionnaire - only omitting demographic and comorbidity questions. Besides the data from the questionnaire, the study assistant will document additional parameters at admission, after surgery and at discharge, encapsulating information on the participant's medical history, the surgery, and aftercare. In Figure 1, the standard protocol items at different points in time are shown.

\section{Randomization and blinding}

The allocation of participants occurs after completion of the patient survey at the day of discharge; thus, during inpatient stay, all eligible patients are treated equally. All participants are stratified into TKR or THR patients and then randomly allocated at a ratio of 1:1 into the intervention group or control group. No further stratifications or corrections are performed. Randomization will be done for each hospital individually due to data security regulations. Figure 2 shows the randomization flow. The study's software provider will be responsible for the randomization algorithm and allocation will only be shown to the study assistant or the physician after discharge of the participant. Hence, during the inpatient stay the hospital staff and the participants are blinded. The participants will stay blinded until the end of the study - not knowing whether they are in the intervention or control group. Frequency of the surveys are not disclosed to patients, neither for the intervention group nor for the control group.

\section{Intervention}

Whereas participants in the control group will be asked to complete only a final follow-up questionnaire 12 months post-discharge, participants in the intervention group have to complete four follow-up questionnaires, at months 1, 3 and 6, and a final follow-up 12 months post-discharge. All follow-up questionnaires are sent via e-mail and are answered electronically by the participants, either on their computers, smartphones or tablets. In case the participants do not possess an e-mail account, e-mails are sent to relatives if agreed upon, and jointly filled out by relative and patient. Results from the questionnaires will be automatically transferred to the study's software and are observable by the 
hospitals' study assistants and physicians. 1, 3, and 6 months results from the aforementioned PROM sets will be screened for critical values or unfavorable developments.

When critical values or developments are detected, the software will consequently alert the study assistants of the corresponding hospitals and an intervention is initiated. Interventions are characterized by three steps: Firstly, the study assistant sends a standardized report to the participant, via post or via e-mail, where critical values and developments are highlighted and a follow-up call is announced. Secondly, the study assistant calls the participants via phone informing them of the critical values, asking permission to forward the PROM results to their outpatient specialist or general practitioner, and recommending a visit to their physician to potentially adjust the treatment. Finally, a report with the participant's results will be forwarded, via post or fax, to the outpatient physician. In the next regular questionnaire, the participants are asked to report changes in their treatment schedule in order to document modifications in the outpatient physicians' treatment, with respect to physiotherapy, medication or surgery.

For health-related quality of life (EQ-5D-5L) and functionality (KOOS-PS, HOOS-PS), critical absolute values and unfavorable development in the recovery paths were defined by an expert consortium of 13 orthopedic physicians, representing all nine participating hospitals. For this definition the Standard Delphi Technique was deployed. The advantage of the Standard Delphi Technique is that it offers a structured process of collecting expert opinions, approaching consensus, without being biased by the domination effect of a few experts in open discussions (16). The physicians separately populated templates for each item of the PROM sets - EQ-5D-5L (for hip and knee), KOOS-PS/HOOS-PS at 1, 3, 6 and 12 months post-surgery - subject to answering the following question: "Which patient value of this particular item would be considered critical at 1,3,6, or 12 months after surgery to schedule an appointment for a follow-up examination?"

Results were collected in one-to-one interviews, consolidated, analyzed and basic statistics were played back to the physicians offering them to adjust their values. Feedback was collected, once again consolidated and results were played back, and discussed in a final joint call. Moreover, it was agreed that neither the EQ-5D-5L nor KOOS-PS/HOOS-PS scores should worsen over time. Defined values have additionally be sanity checked on historic values of patients of two participating study centers, in order to test their sensitivity. For the PROMIS Fatigue and Depression scores, only absolute critical values were set based on existing research - for example, a critical PROMIS Depression score was set to the PHQ-9 classification "moderately severe" depression (17). All critical values are summarized in Table 1.

\section{Data quality enhancement}

Each study assistant is provided with a digital dashboard showing open tasks, critical value alerts, and patients' activity related to receiving and answering follow up questionnaires. Each task is associated 
with a deadline, and a color code reminds the study assistant of open tasks. For the purpose of data completeness, the participants will be reminded by the software to fill in their follow-up questionnaires 1, 2 and 7 days after the initial follow-up questionnaires are sent out. If a participant has not completed the questionnaire after three reminders, the study assistant will call to support him/her.

Moreover, predefined standardized e-mails, guidelines for the follow-ups and intervention calls ensure consistency between study centers. Further, the adherence to the protocol given in the study design was promoted in training on the ground and during a central workshop for all study assistants and physicians.

\section{Hospital administrative data, clinical quality data, and insurance claims data}

After completion of the last participant's questionnaire and only for those who have given consent, hospital administrative data and insurance claims data will be added to the participants' PROM data,. Hospital administrative data is provided by the hospitals' controlling department based on the hospital internal case number of the patient. Insurance claims data is provided for participants who are insured at one of the participating sickness funds and have given consent.

\section{Outcomes}

The overall aim of the study is to test the cost-effectiveness of PROMs for early identification and therefore timely treatment of post-surgical complications and/or the health-related quality decreasing developments. The primary endpoint of the study is to investigate cost-effectiveness, thus the optimization of outcome quality compared to the resource utilization of the designed intervention across the pathway. Outcome quality is defined as a composite measure of PROMs and clinical outcome measures such as re-operations. Utilized resources are direct and indirect follow-up health care cost and the cost of implementing the designed intervention.

Secondary outcomes include the improvement of the patients' functionality reflected by the KOOSPS/HOOS-PS, of medium to long-term health-related quality of life evaluated by the EQ-5D-5L, of pain in knee, hip and lower back, of satisfaction with treatment outcome (measured through selected questions from the PEQ questionnaire) and of clinical outcome measures like post-operative revision and re-operation rates. Furthermore, these analyses will be repeated with subgroups. Specific analyses will be carried out to estimate the impact of enhanced recovery programs or the choice of a specific implant on patient-reported outcome quality, for example.

\section{Statistical analysis}

Data will be provided in electronic formats. A statistical analysis program will be used to run tests and analyses. For the planned statistical analyses, it has to be distinguished between the primary and the secondary outcome.

For primary outcome - the cost-effectiveness of using PROMs in TKR/THR - a cost-effectiveness analysis (CEA) will be conducted. In the CEA, alternatives will be evaluated by costs and consequences 
(quality improvement) and mapped on a cost-effectiveness plane (18) and thus, create support for later policy recommendations. For costs, the cross-sectoral sickness funds' claims data on patient-level for direct and indirect follow-up costs as well as the cost of the intervention are taken into account to calculate incremental cost. For the patients' overall health status, a composite measure is used. Results for each patients' quality indicator are gathered, standardized and then combined into a quality index. This composite measure is a formative construct as it is expected that the different quality indicators will correlate (19). In order to test for the robustness of the results, it is planned to perform scenario and/or probabilistic sensitivity analyses.

For the secondary endpoints, we will mostly apply multiple regressions using the differences in the quality indicator outcomes and improvements between intervention and control group. Several subgroup-level analyses for distinct and relevant patient cohorts will be performed. Beside others, subgroups will cover age, sex, education, mobilization, comorbidity, type of prosthesis

\section{Data management}

The collection, storage and processing of personal data in this study is carried out in accordance with the applicable data protection regulations of the federal states in connection with the European General Data Protection Regulation (GDPR) and taking into account the specific provisions of the German Social Code, Tenth Book (SGB X). During the study, all data recorded electronically or in the hospital will be stored at the respective hospital servers and can only be accessed or decrypted by authorized users in the hospital environment. Data will be transferred to a secured personalized access SFTP-Server of the evaluating institute. Every authorized user is assigned to a separate area on the server where transport-encrypted (AES-256) data can be stored and retrieved.

All transferred data will be completely pseudonymized. Consequently, to match primary patient data to hospital administrative data and insurance claims data a pseudonym will be created by the study's software following UUID Standard (Universally Unique Identifier) and is added to the patient data. The pseudonym will then be added to the hospital administrative data and insurance claims data. All other identification data will be deleted before it is transferred to the researching institute. Pseudonymized data will be stored for two years at the researching institute (TU Berlin) - and stored at study centers for ten years after the study completion to ensure further evaluation of study results. This is compliant with the recommendation for good practice for secondary data analysis (20).

\section{Study management}

Study management is led by the project team at the TU Berlin. The overall project team consists of the responsible person for the study design, members from the two participating German sickness funds, the technical provider, and all study assistants. The team meets on a regular one-month basis by teleconference to discuss the progress and solve uprising problems. Regular one-on-one calls are conducted between the TU Berlin project team and study assistants to ensure enrollment in line with 
the study protocol. Additionally, on-site visits are planned every 3-4 months. During these sessions, the study management will monitor adherence to the study design.

Furthermore, intermediate reports with statistics on recruitment and follow-up rates are sent to the study management monthly. Regular status updates are sent to the study sponsor. Intermediate reports contain only aggregated data without participant specific data. After 6 and 12 months, patient data sets will be extracted from the software to check data quality and consistency. Moreover, payouts to the hospitals are linked to patient recruitment rates (based on their internally projected yearly case volume) and follow-up completion rates. Therefore, study assistants need to export statistics at given points in time to receive full compensation.

Due to the character of the study, there are no adverse effects expected, but unfavorable developments will rather initiate an intervention and be part of the study design. 


\section{Discussion}

Recent studies have shown the value of PROMs in monitoring patients' recovery paths in oncologic settings $(12,13)$, as well as their effectiveness in measuring patient-relevant quality after TKR/THR (7, 8). However, according to our knowledge, no randomized controlled trial exists that examines outcome improvement and cost-effectiveness of monitoring and intervening in case of critical recovery paths by the help of PROMs.

\section{Insights}

Depending on a positive and cost-effective impact of the intervention, a further aim is to evaluate the feasibility of implementing regular and digitized PROM-based communication into standard care. Furthermore, results from this study can be leveraged for the definition and implementation of in German recently discussed selective contracts between sickness funds and health providers (21). The setup phase of our study revealed several insights.

First, digitized PROMs can be a benefit but also a challenge to hospitals as well as patients. Collection of PROMs on paper has unfortunately been not effective with low follow-up return rates and a burden of additional documentation work for hospitals. Digitized PROMs promise higher return rates and significantly reduced documentation work. Nevertheless, hospital IT departments need to be collaborative, personnel needs to be trained to familiarize patients with digital tools.

Second, structural changes are needed to enable seamless implementation of the study design. The effectiveness of PROMs requires acceptance by physicians as well as hospital management. It could be observed, that a move towards patient centeredness was generally perceived as very positive by physicians and study assistants. Nevertheless, resources are required for personnel who familiarize patients with PROMs and their relevance in the aftercare and who compile the data. This relationship between study assistant and patient is the central lever for data quality and completeness. Moreover, effective communication channels between hospitals and outpatient physicians and patients are key for the best possible recovery pathway. Often, this communication is disrupted due to the boundaries between the siloed in-and outpatient sector in the German health care system.

Third, data privacy and security regulation were a challenging factor in the setup of the study. New data security regulations complicate the communication between hospitals, patients and outpatient physicians. A common nation-wide health data platform for collecting data and for communicating results could be an interesting idea for further discussion.

Lastly, in Germany, there is a diverse landscape of hospitals, reaching from full-service providers to specialized hospitals and specialist outpatient clinics. The diversity of the set of hospitals in the study sample already indicates that a standardized "one fits all" process will not work. The collected insights in hindsight can be leveraged for further analysis to define the degree of standardization in the approach. 


\section{Limitations}

There is a number of limitations to the study at hand. Firstly, due to data security regulations and complexity reasons, outpatient physicians are not formally part of the study. Unfortunately, we can only advise but not oblige the outpatient physicians to use the participants' critical PROM results in their further treatment plan. We try to convince them of the study relevance by sending information material, directing to our study website, and thorough conversation with the participant. Secondly, patients without direct or indirect access to an e-mail address cannot be included in the study. We hope to minimize this effect by involving their relatives. Thirdly, there is no empirical evidence on critical PROM values at specific points in time yet. We have tried to overcome this limitation with the formation of an expert consortium and a real data check. Nevertheless, the defined critical values might be over/underestimated, thus including participants with non-critical recovery paths or missing participants with critical recovery paths. Fourthly, participants in the intervention group might have a learning curve when responding to the follow-up questionnaires, deliberately avoiding interventions. From our point of view, this effect is unlikely as the participant does not profit from avoiding interventions. Finally, differences in personnel, organization and infrastructure between study centers might influence the patient pathway during the hospital stay - despite adherence to the study protocol.

\section{Dissemination}

The results of this study will be submitted for publication in relevant journals, presented in relevant conferences and used in the political discourse. Results will play a role in conversations with the sickness funds and the Federal Joint Committee for developing a German-wide implementation. Participating hospitals will be included in the publications and have the right for publication of their data. All results will be on an aggregated basis and cannot be connected to individual participants.

\section{Trial status}

The current protocol is version 1, dated January 15th 2019. Recruitment of patients began, dependent on the study center between October 1st and January 1st 2020 and will approximately end December 31 st 2020. The trial is still open at the time of this study protocol submission. The study is expected to run until March 31st, 2023. 


\title{
Declarations
}

\begin{abstract}
Abbreviations
CEA $=$ Cost-effectiveness analysis; DRKS $=$ Deutsches Register für klinische Studien (German Clinical Trails Register); ICHOM = International Consortium for Health Outcomes Measurement; GDPR = General Data Protection Regulation; PEQ = Patient Experience Questionnaire; PROMs = Patient reported outcome measures; RCT $=$ Randomized controlled trial; $\mathrm{THR}=$ Total hip replacement; TKR $=$ Total knee replacement; TU Berlin $=$ Technical University Berlin
\end{abstract}

\section{Acknowledgements}

We thank the orthopedic physicians and study assistants involved for their engagement in the design and the study itself. We thank all physicians who were part of the expert consortium for supporting in defining the critical thresholds. We thank the study centers for their contributions to realize this study, Charité, DIAKOVERE Annastift, Helios ENDO-Klinik Hamburg GmbH, RoMed Kliniken Prien a. Chiemsee, Sana Kliniken Sommerfeld, Schön Klinik Hamburg Eilbek, Schön Klinik Neustadt, VAMED Ostseeklinik Damp GmbH, Waldkliniken Eisenberg GmbH. We thank HRTBT Medical $\mathrm{GmbH}$ for technically implementing the study design. We thank Thorben Breitkreuz and Thomas Grobe from the aQua institute for supporting with statistical analyses, power calculations and for proofreading the document. We thank the sickness funds BARMER and BKK Dachverband for their project partnership. We thank Justus Vogel and Dr. Jens Deerberg-Wittram for their valuable input and support during the grant application process. We acknowledge support by the Open Access Publication Fund of TU Berlin.

\section{Funding}

The study is funded by the Innovation Fund of the German Federal Joint Committee (G-BA) in the stream 'Care models with comprehensive and measurable results and process responsibility' under the funding code 01NVF18016. The funding period is set between April 1st, 2019 and March 31st, 2023. Neither was the funding body actively involved in the study design nor is it in the data collection, analysis or interpretation of the results, but rather acted and will act as a reviewing and monitoring body.

\section{Consent for publication}

Material in this manuscript is not subject to publication restrictions.

\section{Availability of data and materials}

Datasets generated and collected by this study will not be publicly available. Analyses of the collected data will only be granted to researching institute, the evaluating institute and the participating hospitals.

\section{Authors' contributions}

DK, LO, CP and AG were responsible for the study design and methodology. LO is leading the project administration, whereas project supervision was given be AG, RB and CP. DK drafted the first version of this document. LO, CP, RB and AG provided valuable feedback on the manuscript. All authors provided critical intellectual input to the manuscript and read and approved the final version of the manuscript, agreeing to be accountable for all aspects of the work.

\section{Ethics approval and consent to participate}

The study was approved by the lead Charité Ethics Committee, Berlin (EA4/169/19). The other responsible ethical review committees assented (Medical Association Hamburg, Medical Association Schleswig-Holstein, Hannover Medical School, Friedrich-Schiller University Jena, and the Medical Association Brandenburg. The study is registered at the German Clinical Trials Register (DRKS) under 
DRKS00019916. All potentially eligible participants will be approached to offer their informed consent to participate in the study. Additionally, they can accept to release their hospital administrative data in an optional consent form. For patients insured by participating sickness funds a third consent form is handed out to ask for permission to process their insurance claims data at the end of the study period. Patients will be free to withdraw from either of the three consents without stating a reason. If the patients withdraw from the consent for participation then all their data will be deleted.

\section{Competing interests}

$\mathrm{CP}$ is full-time contracted with Stryker Corporation, a medical technology company, that also produces knee and hip implants. The authors declare that they have no further competing interests. 


\section{Appendices}

Figure 1: Standard protocol items - PROMoting Quality

\begin{tabular}{|c|c|c|c|c|c|c|c|c|c|}
\hline & Enrolment & \multicolumn{2}{|c|}{ Baseline } & Allocation & \multicolumn{3}{|c|}{ Follow-ups } & \multicolumn{2}{|c|}{ Close-out } \\
\hline Timepoint & $\begin{array}{c}\text { Pre- } \\
\text { admission }\end{array}$ & Admission & Discharge & Discharge & $\begin{array}{c}\text { Month } \\
1\end{array}$ & $\begin{array}{c}\text { Month } \\
3\end{array}$ & $\begin{array}{c}\text { Month } \\
6\end{array}$ & $\begin{array}{c}\text { Month } \\
12\end{array}$ & $\begin{array}{c}\text { October } \\
\text { '21 }\end{array}$ \\
\hline \multicolumn{10}{|l|}{ ENROLMENT: } \\
\hline Eligibility screen & $\mathrm{X}$ & & & & & & & & \\
\hline Informed consent & $\mathrm{X}$ & & & & & & & & \\
\hline Contact information & $\mathrm{X}$ & & $\mathrm{X}$ & & & & & & \\
\hline Baseline Questionnaire & & $\mathrm{X}$ & $\mathrm{X}$ & & & & & & \\
\hline Allocation & & & & $\mathrm{X}$ & & & & & \\
\hline \multicolumn{10}{|c|}{ INTERVENTIONS/COLLECTION MOMENTS: } \\
\hline Intervention Group & $\mathrm{X}$ & $\mathrm{X}$ & $\mathrm{X}$ & $\mathrm{X}$ & $\mathrm{X}$ & $\mathrm{X}$ & $\mathrm{X}$ & $\mathrm{X}$ & $\mathrm{X} * / * *$ \\
\hline Control Group & $\mathrm{X}$ & $\mathrm{X}$ & $\mathrm{X}$ & $\mathrm{X}$ & & & & $\mathrm{X}$ & $\mathrm{X} * / * *$ \\
\hline \multicolumn{10}{|l|}{ ASSESSMENTS: } \\
\hline Comorbidity data & & $\mathrm{X}$ & & & & & & & \\
\hline $\begin{array}{c}\text { Clinical data } \\
\text { (surgeon report, admission } \\
\text { interview) }\end{array}$ & & $\mathrm{X}$ & $\mathrm{X}$ & & & & & & \\
\hline $\begin{array}{c}\text { PROMS (EQ-5D-5L, KOOS- } \\
\text { PS/HOOS-PS, PROMIS) }\end{array}$ & & $\mathrm{X}$ & $\mathrm{X}$ & & $\mathrm{X}$ & $\mathrm{X}$ & $\mathrm{X}$ & $\mathrm{X}$ & \\
\hline $\begin{array}{c}\text { Clinical Outcomes (revisions, } \\
\text { re-operation) }\end{array}$ & & & & & & & & & $\mathrm{X}$ \\
\hline $\begin{array}{l}\text { Treatment data (changes in } \\
\text { treatment schedule) }\end{array}$ & & & & & $\mathrm{X}$ & $\mathrm{X}$ & $\mathrm{X}$ & $\mathrm{X}$ & \\
\hline Patient satisfaction data & & & & & $\mathrm{X}$ & $\mathrm{X}$ & $\mathrm{X}$ & $\mathrm{X}$ & \\
\hline Hospital administrative data & & & & & & & & & $\mathrm{X}^{*}$ \\
\hline Insurance claims data & & & & & & & & & $\mathrm{X}^{* *}$ \\
\hline
\end{tabular}

* If patient has given consent to process hospital administrative data; ** if patient is insured in participating sickness funds and has given consent to process insurance claims data 


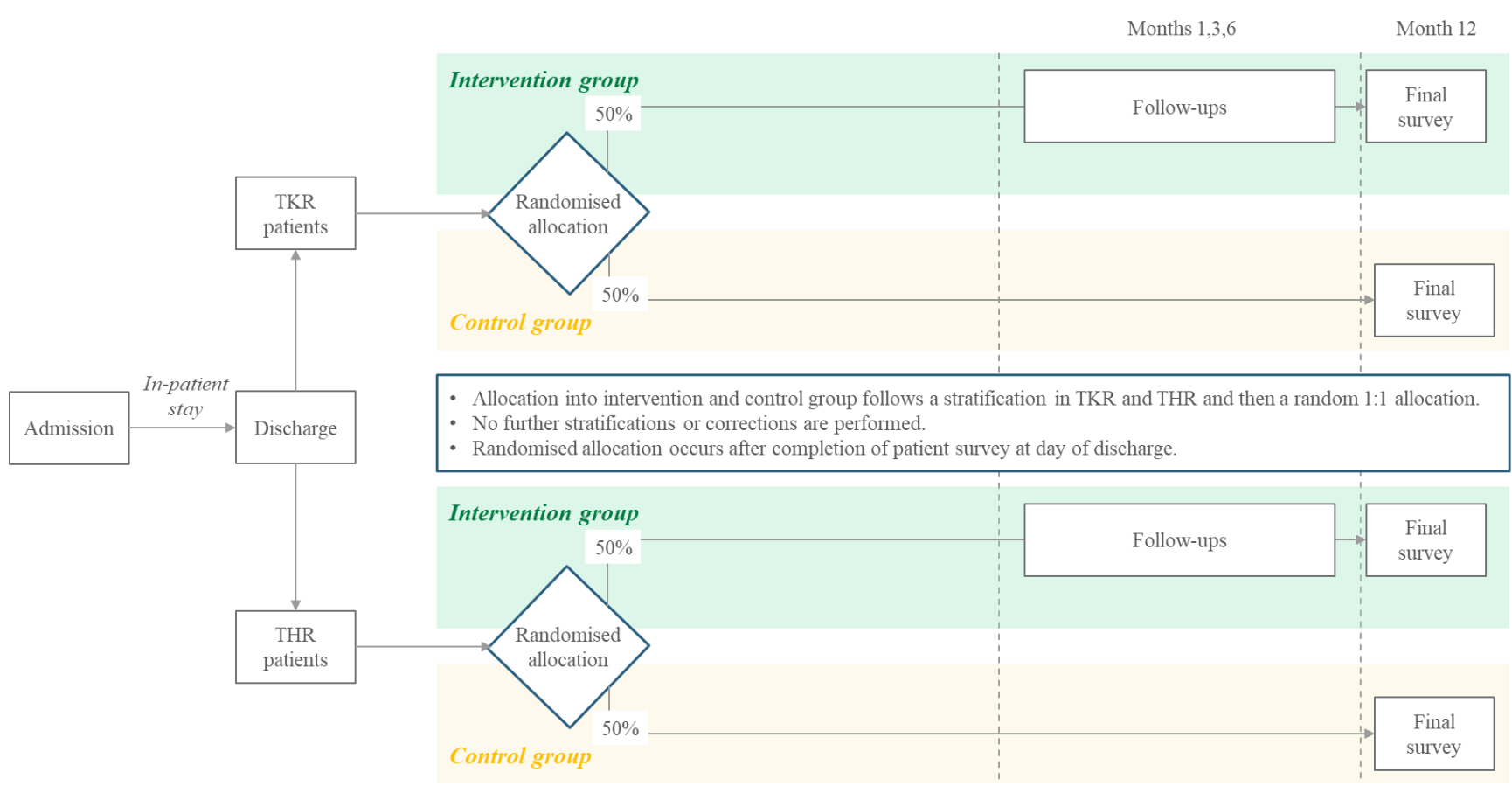

Table 1: Summary of critical values

\begin{tabular}{|l|r|l|r|r|}
\hline PROM Sets & Month 1 & Month 3 & Month 6 & $\begin{array}{l}\text { Critical } \\
\text { developments* }\end{array}$ \\
\hline EQ-5D-5L hip & 0.37 & 0.64 & 0.74 & Yes \\
\hline HOOS-PS & 53.00 & 36.30 & 27.70 & Yes \\
\hline EQ-5D-5L knee & 0.36 & 0.51 & 0.70 & Yes \\
\hline KOOS-PS & 51.60 & 43.60 & 33.60 & Yes \\
\hline PROMIS Depression & 65.80 & 65.80 & 65.80 & No \\
\hline PROMIS Fatigue & 69.00 & 69.00 & 69.00 & No \\
\hline
\end{tabular}

* Alerts at critical development defined as worsening score compared to previous questionnaire

\section{Additional File 1: Informed consent}

See additional file "Informed Consent"

Additional File 2: SPIRIT Check-List

See additional file "SPIRIT Check-List" 
Literature Cited

1. Forschungsdatenzentren der Statistischen Ämter des Bundes und der Länder.

Fallpauschalenbezogene Krankenhausstatistik (DRG-Statistik) 2016, On-Site, Version 0.

2. Pabinger C, Geissler A. Utilization rates of hip arthroplasty in OECD countries. Osteoarthr Cartil 2014; 22(6):734-41.

3. Pabinger C, Lothaller H, Geissler A. Utilization rates of knee-arthroplasty in OECD countries. Osteoarthr Cartil 2015; 23(10):1664-73.

4. Pabinger C, Lothaller H, Portner N, Geissler A. Projections of hip arthroplasty in OECD countries up to 2050. Hip Int 2018; 28(5):498-506.

5. Pross C, Geissler A, Busse R. Measuring, Reporting, and Rewarding Quality of Care in 5 Nations: 5 Policy Levers to Enhance Hospital Quality Accountability. Milbank Q 2017; 95(1):136-83.

6. Valderas JM, Kotzeva A, Espallargues M, Guyatt G, Ferrans CE, Halyard MY et al. The impact of measuring patient-reported outcomes in clinical practice: a systematic review of the literature. Qual Life Res 2008; 17(2):179-93.

7. Homm R. Patient-reported Outcomes (PROs) bei Hüft-und Kniegelenkersatz. In: Klauber J, Dormann F, editors. Qualitätsmonitor 2017. Berlin: Medizinisch Wissenschaftliche Verlagsgesellschaft; 2017.

8. Gutacker N, Siciliani L, Moscelli G, Gravelle H. Choice of hospital: Which type of quality matters? J Health Econ 2016; 50:230-46.

9. Baker PN, Deehan DJ, Lees D, Jameson S, Avery PJ, Gregg PJ et al. The effect of surgical factors on early patient-reported outcome measures (PROMS) following total knee replacement. J Bone Joint Surg Br 2012; 94(8):1058-66.

10. İmren Y, Dedeoğlu SS, Çakar M, Çabuk H, Bayraktar TO, Gürbüz H. Infrapatellar Fat Pad Excision during Total Knee Arthroplasty Did Not Alter the Patellar Tendon Length: A 5-Year Follow-Up Study. J Knee Surg 2017; 30(5):479-83.

11. Beschluss des Gemeinsamen Bundesausschusses über die Abnahme des Entwurfs der Konzeptskizze der Institution nach § 137a SGB V für ein Qualitätssicherungsverfahren für Entlassungsmanagement; 2017.

12. Basch E, Deal AM, Dueck AC, Scher HI, Kris MG, Hudis C et al. Overall Survival Results of a Trial Assessing Patient-Reported Outcomes for Symptom Monitoring During Routine Cancer Treatment. JAMA 2017; 318(2):197-8. 
13. Basch E, Deal AM, Kris MG, Scher HI, Hudis CA, Sabbatini P et al. Symptom Monitoring With Patient-Reported Outcomes During Routine Cancer Treatment: A Randomized Controlled Trial. J Clin Oncol 2016; 34(6):557-65.

14. Busse R, Blümel M, Knieps F, Bärnighausen T. Statutory health insurance in Germany: a health system shaped by 135 years of solidarity, self-governance, and competition. The Lancet 2017; 390(10097):882-97.

15. Singh JA, Lewallen DG. Depression in primary TKA and higher medical comorbidities in revision TKA are associated with suboptimal subjective improvement in knee function. BMC Musculoskelet Disord 2014; 15:127.

16. Jairath N, Weinstein J. The Delphi methodology (Part one): A useful administrative approach. Can J Nurs Adm 1994; 7(3):29-42.

17. Choi SW, Schalet B, Cook KF, Cella D. Establishing a common metric for depressive symptoms: linking the BDI-II, CES-D, and PHQ-9 to PROMIS depression. Psychol Assess 2014; 26(2):513-27.

18. Rudmik L, Drummond M. Health economic evaluation: important principles and methodology. Laryngoscope 2013; 123(6):1341-7.

19. Shwartz M, Restuccia JD, Rosen AK. Composite Measures of Health Care Provider Performance: A Description of Approaches. Milbank Q 2015; 93(4):788-825.

20. Swart E, Gothe H, Geyer S, Jaunzeme J, Maier B, Grobe TG et al. Gute Praxis Sekundärdatenanalyse (GPS): Leitlinien und Empfehlungen. Gesundheitswesen 2015; 77(2):120-6.

21. Qualitätsverträge nach $\S 110$ a SGB V - Evaluationskonzept zur Untersuchung der Entwicklung der Versorgungsqualität gemäß § 136b Abs. 8 SGB V; 2017. 
Figures

\begin{tabular}{|c|c|c|c|c|c|c|c|c|c|}
\hline & Enrolment & \multicolumn{2}{|c|}{ Baseline } & Allocation & \multicolumn{3}{|c|}{ Follow-ups } & \multicolumn{2}{|c|}{ Close-out } \\
\hline Timepoint & $\begin{array}{c}\text { Pre- } \\
\text { admission }\end{array}$ & Admission & Discharge & Discharge & $\begin{array}{c}\text { Month } \\
1\end{array}$ & $\begin{array}{c}\text { Month } \\
3\end{array}$ & $\begin{array}{c}\text { Month } \\
6\end{array}$ & $\begin{array}{c}\text { Month } \\
12\end{array}$ & $\begin{array}{c}\text { October } \\
\text { '21 }\end{array}$ \\
\hline \multicolumn{10}{|l|}{ ENROLMENT: } \\
\hline Eligibility screen & $\mathrm{X}$ & & & & & & & & \\
\hline Informed consent & $\mathrm{X}$ & & & & & & & & \\
\hline Contact information & $\mathrm{X}$ & & $\mathrm{X}$ & & & & & & \\
\hline Baseline Questionnaire & & $\mathrm{X}$ & $\mathrm{X}$ & & & & & & \\
\hline Allocation & & & & $\mathrm{X}$ & & & & & \\
\hline \multicolumn{10}{|c|}{ INTERVENTIONS/COLLECTION MOMENTS: } \\
\hline Intervention Group & $\mathrm{X}$ & $\mathrm{X}$ & $\mathrm{X}$ & $\mathrm{X}$ & $\mathrm{X}$ & $\mathrm{X}$ & $\mathrm{X}$ & $\mathrm{X}$ & $\mathrm{X}^{* / * *}$ \\
\hline Control Group & $\mathrm{X}$ & $\mathrm{X}$ & $\mathrm{X}$ & $\mathrm{X}$ & & & & $\mathrm{X}$ & $\mathrm{X}^{* / * * *}$ \\
\hline \multicolumn{10}{|l|}{ ASSESSMENTS: } \\
\hline Comorbidity data & & $\mathrm{X}$ & & & & & & & \\
\hline $\begin{array}{c}\text { Clinical data } \\
\text { (surgeon report, admission } \\
\text { interview) }\end{array}$ & & $\mathrm{X}$ & $\mathrm{X}$ & & & & & & \\
\hline $\begin{array}{c}\text { PROMS (EQ-5D-5L, KOOS- } \\
\text { PS/HOOS-PS, PROMIS) }\end{array}$ & & $\mathrm{X}$ & $\mathrm{X}$ & & $\mathrm{X}$ & $\mathrm{X}$ & $\mathrm{X}$ & $\mathrm{X}$ & \\
\hline $\begin{array}{c}\text { Clinical Outcomes (revisions, } \\
\text { re-operation) }\end{array}$ & & & & & & & & & $\mathrm{X}$ \\
\hline $\begin{array}{c}\text { Treatment data (changes in } \\
\text { treatment schedule) }\end{array}$ & & & & & $\mathrm{X}$ & $\mathrm{X}$ & $\mathrm{X}$ & $\mathrm{X}$ & \\
\hline Patient satisfaction data & & & & & $\mathrm{X}$ & $\mathrm{X}$ & $\mathrm{X}$ & $\mathrm{X}$ & \\
\hline Hospital administrative data & & & & & & & & & $\mathrm{X}^{*}$ \\
\hline Insurance claims data & & & & & & & & & $\mathrm{X}^{* *}$ \\
\hline
\end{tabular}

* If patient has given consent to process hospital administrative data; ${ }^{* *}$ if patient is insured in participating sickness funds and has given consent to process insurance claims data

Figure 1

Standard protocol items - PROMoting Quality 


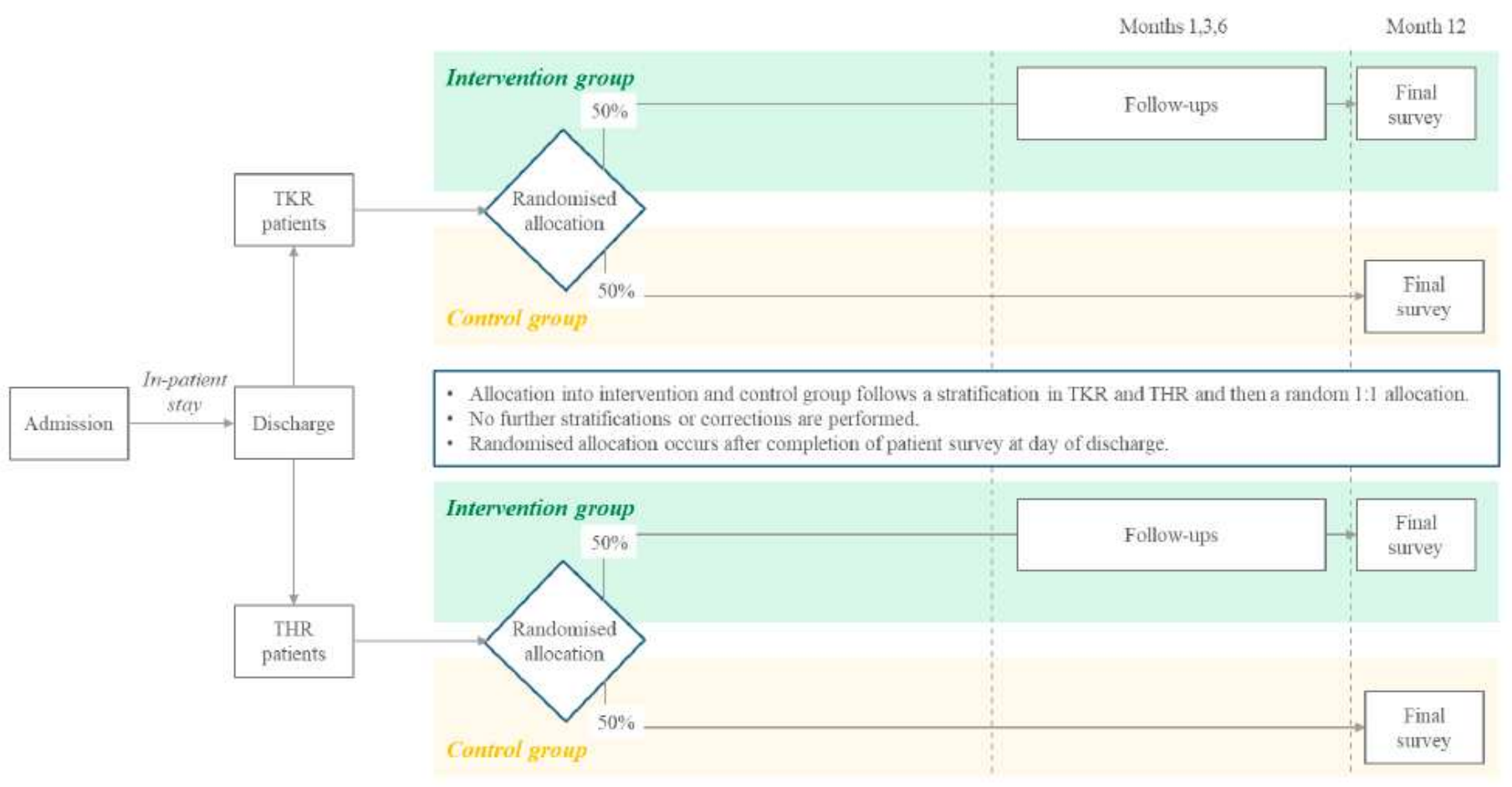

Figure 2

Randomization process

\section{Supplementary Files}

This is a list of supplementary files associated with this preprint. Click to download.

- AdditionalFile2SPIRITchecklist.pdf

- AdditionalFile1InformedConsent.pdf 